\title{
ON THE SUBCENTRAL AUTOMORPHISMS OF FINITE GROUPS
}

\author{
PARISA SEIFIZADEH and MOHAMMAD MEHDI NASRABADI*
}

\begin{abstract}
Let $G$ be a group and let $M$ be a characteristic subgroup of $G$. We denote by $\operatorname{Aut}_{M}^{M}(G)$ the set of all automorphisms of $G$ which centralize $G / M$ and $M$. In this paper, we give necessary and sufficient conditions for the equality of $\operatorname{Aut}_{M}^{M}(G)$ with $\operatorname{Aut}^{M}(G)$ and $C_{\mathrm{Aut}_{M}^{M}(G)}(Z(G))$.
\end{abstract}

MSC 2010. Primary: 20D45; Secondary: 20D15.

Key words. Characteristic subgroup, finite $p$-groups, Frattini subgroup, inner automorphism, subcentral automorphism.

\section{REFERENCES}

[1] J.E. Adney and T. Yen, Automorphisms of a p-group, Illinois J. Math., 9 (1965), 137 143.

[2] Z. Azhdari and M. Akhavan-Malayeri, On automorphisms fixing certain groups, J. Algebra Appl., 12 (2013), 1250163-1-17.

[3] R.D. Carmichael, Groups of finite order, Dover Publications, New York, 1965.

[4] M.J. Curran and D.J. McCaughan, Central automorphisms that are almost inner, Comm. Algebra, 29 (2001), 2081-2087.

[5] M.J. Curran and D.J. McCaughan, Finite groups with central automorphism group of minimal order, Math. Proc. R. Ir. Acad., 104A (2004), 223-229.

[6] R.G. Ghumde and S.H. Ghate, Group of automorphisms preserving cosets of a central charactristic subgroup and related results, Acta Math. Univ. Comenian., 2 (2016), 181189.

[7] Z. Kaboutari Farimani and M.M. Nasrabadi, On absolute central automorphisms fixing the center elementwise, J. Algebr. Syst., 2 (2016), 127-131.

[8] M. Shabani Attar, Finite p-groups in which each central automorphism fixes centre elementwise, Comm. Algebra, 40 (2012), 1096-1102.

Received March 17, 2019

Accepted July 21, 2019

The authors thank the referee for his helpful comments and suggestions

*Corresponding author

DOI: $10.24193 /$ mathcluj.2020.1.09 
University of Birjand

Department of Mathematics

Birjand, Iran

E-mail: paris.seifizadeh@birjand.ac.ir

E-mail: jparis.seifizade@gmail.com

E-mail: mnasrabadi@birjand.ac.ir 